\title{
Assessment of Effect of Implant Angulation and Implant Number on the Dimensional Accuracy of Definitive Casts
}

\author{
Gurpreet Kaur ${ }^{1}$, Parvinder S Baweja ${ }^{2}$, Ravinder S Saini ${ }^{3}$, Ish Paul Singh ${ }^{4}$, Tanvi Sharma ${ }^{5}$, Simarpreet Singh ${ }^{6}$
}

\begin{abstract}
Aim: To analyze the impact of implant angulation and implant number on the dimensional accuracy of definitive casts.

Materials and methods: Seven definitive casts with implant analogs placed in a triangular pattern were made from dental stone. The groups were as follows: group 1 (control group) - all implant analogs perpendicular to the plane of the cast; the center implant analog (implant 2) being at $90^{\circ}$ to the plane of the cast in all groups and implant analogs number 1 and 3 arranged in $5^{\circ}, 10^{\circ}$, or $15^{\circ}$ divergence from or convergence to the center implant (implant number 2). Three open-tray impressions of definitive casts were made in each group. Impressions were poured in type IV dental stone. Coordinates in the three planes were measured at the implant analog top surface and the base of the cast using a fine tip measuring stylus. The data were aligned and the angular differences between implant analog vectors from definitive and duplicate casts were calculated in degrees.

Results: The impact of implant number on the dimensional accuracy of definitive casts was significant whereas for implant angulation it was nonsignificant. The correlation of angulation and the number of the implant did not show an interpretable pattern. The precision of duplicate casts (compared to definitive casts) is not affected by the combined interaction of implant number and implant angulation.

Conclusion: The close proximity of implant angulation toward a right-angled direction results in higher precision of implant. In the impression, there is direct picking from the impression copings which decreases the discrepancies in implant angulation on impression and master cast. The interaction of implant angulation and the number shows a noninterpretable pattern.

Clinical significance: Dimensionally accurate implant impressions can be made by using an open tray technique for three implants angled up to $15^{\circ}$.

Keywords: Dimensional accuracy, Implant angulation, Implant impressions, Implant number, Open tray technique.

The Journal of Contemporary Dental Practice (2021): 10.5005/jp-journals-10024-3053
\end{abstract}

\section{INTRODUCTION}

Major advancements have been made in the rehabilitation of patients with lost teeth. One of the most promising developments has been the treatment with root-form endosseous osseointegrated implants. ${ }^{1}$ Data from past literature quote a number of researchers who favor the long-term prognosis of the outcome of dental implants. Despite a high success rate of dental implant therapy (more than 97\%), the occurrence of failure and complication in dental implant therapy is not uncommon. Most of these complications are due to a faulty surgical or a prosthodontic technique. ${ }^{2-5}$ Imprecise location of the dental implant in the primary cast leads to significant technical problems and makes it extremely difficult to construct an ideal-like prosthesis. As a result, a series of biomechanical complications can occur including screw loosening, loss of alveolar bone, and ceramic veneer fracture. These problems further predispose to enhanced stress-related problems at dental implant-bone-related interface or within the prosthetic component. Several factors affect the accuracy and precision of the master cast. This includes impression technique, tray type used for impression making, dental stone manipulation technique, and compatibility of the cast with impression material. Any deviation from the above-mentioned parameters could create an inevitable error. Placement of several implants in a single patient with dissimilar angulations could create distortional changes of the impression material while retrieval. ${ }^{1-3}$

The location of dental implants is detailed and relocated on a working stone cast with the purpose of developing implantsupported prosthesis. The accurate transfer of each implant
${ }^{1}$ Department of Prosthodontics, Baba Jaswant Singh Dental College, Hospital and Research Institute, Ludhiana, Punjab, India

${ }^{2}$ Department of Conservative Dentistry, Baba Jaswant Singh Dental College, Hospital and Research Institute, Ludhiana, Punjab, India

${ }^{3}$ Department of Prosthodontics, COAMS, King Khalid University, Abha, Kingdom of Saudi Arabia

${ }^{4}$ Department of Oral Pathology, Baba Jaswant Singh Dental College, Hospital and Research Institute, Ludhiana, Punjab, India

${ }^{5}$ Intern, Baba Jaswant Singh Dental College, Hospital and Research Institute, Ludhiana, Punjab, India

${ }^{6}$ Department of Oral Surgery, Baba Jaswant Singh Dental College, Hospital and Research Institute, Ludhiana, Punjab, India

Corresponding Author: Gurpreet Kaur, Department of Prosthodontics, Baba Jaswant Singh Dental College, Hospital and Research Institute, Ludhiana, Punjab, India, Phone: +91 9915759100, e-mail: doctorgurpreet@gmail.com

How to cite this article: Kaur G, Baweja PS, Saini RS, et al. Assessment of Effect of Implant Angulation and Implant Number on the Dimensional Accuracy of Definitive Casts. J Contemp Dent Pract 2021;22(4):394-399.

Source of support: Nil

Conflict of interest: None

location is of utmost necessity for the precision in design and fit of implant-supported prosthesis. The major objective of fabricating implant-supported restorations is the production of superstructures that exhibit passive fit when connected to multiple abutments. ${ }^{2}$ Imprecise superstructure fit leads to differential consequences 
that interrupt the utility of dental implants. ${ }^{3-6}$ One of the main requirements for obtaining a passive fit is the making of an accurate impression. ${ }^{7}$ Two basic impression techniques are commonly used for the transfer of implant positions from an intraoral situation to a working cast: the open tray technique and the closed tray technique. ${ }^{8}$ The technique of open tray encompasses implant fastening to an impression handling with a screw and through impression tray's opening cut. After setting off the material, the screw is released and detachment of the tray is done along with retention of coping inside the impression. ${ }^{7}$ In the closed tray technique, detachment of the tray does not occur after the removal of impression. ${ }^{6,7}$ The main purpose of a multi-implant impression is to record and transfer the relationship between implant abutments and implants and to reproduce this relationship as accurately as possible. ${ }^{7-9}$ Hence, the present study was undertaken for assessing the impact of implant angulation and implant number on the dimensional precision of implant definitive casts.

\section{Materials and Methods}

The present study was conducted in the Department of Prosthodontics in Sri Guru Ram Das Institute of Dental Sciences and Research, Sri Amritsar. Seven definitive casts were made by manipulating type IV gypsum (Zhermack) according to the manufacturer's instructions and pouring into a $3.5 \mathrm{~cm} \times 1.75 \mathrm{~cm}$ hemispherically preformed mold (Fig. 1A) using the vibrator (Confident). The casts were retrieved from the mold after setting off the material.

A plane was created on all of the definitive casts with three implants (implant no. 1, 2, and 3) placed in a triangular pattern (Fig. 1B). The distance between implant numbers 1 and 2 was $11 \mathrm{~mm}$, between 2 and 3 was $11 \mathrm{~mm}$, and between 3 and 1 was $15 \mathrm{~mm}$. Three points (each of diameter $1.6 \mathrm{~mm}$ and depth $0.5 \mathrm{~mm}$ ) were punched at three widely separated points on the base of the cast each at a distance of $2 \mathrm{~mm}$ from the outer surface of the cast (Fig. 1C). The holes were plunged cut in the casts by holding in a vertical milling machine (Mikron, Switzerland). The holes matched the diameter and the depth of the implant analogs $(10 \times 3.75 \mathrm{~mm}$, Life Care Devices Pvt. Ltd.,) and degree of convergence or divergence of implant no. 1 and 3 from the center implant (implant no. 2-perpendicular to the cast base in all seven definitive casts).

The holes for these implants were milled at $5^{\circ}, 10^{\circ}$, and $15^{\circ}$ of divergence or convergence as per the following groups (Fig. 1D):

Group 1 (control group): All three implants are perpendicular to the plane of the cast.

Group 2: Implant numbers 1 and 3 are at $5^{\circ}$ convergence to the center implant (implant number 2).

Group 3: Implant numbers 1 and 3 are at $5^{\circ}$ divergence to the center implant (implant number 2).
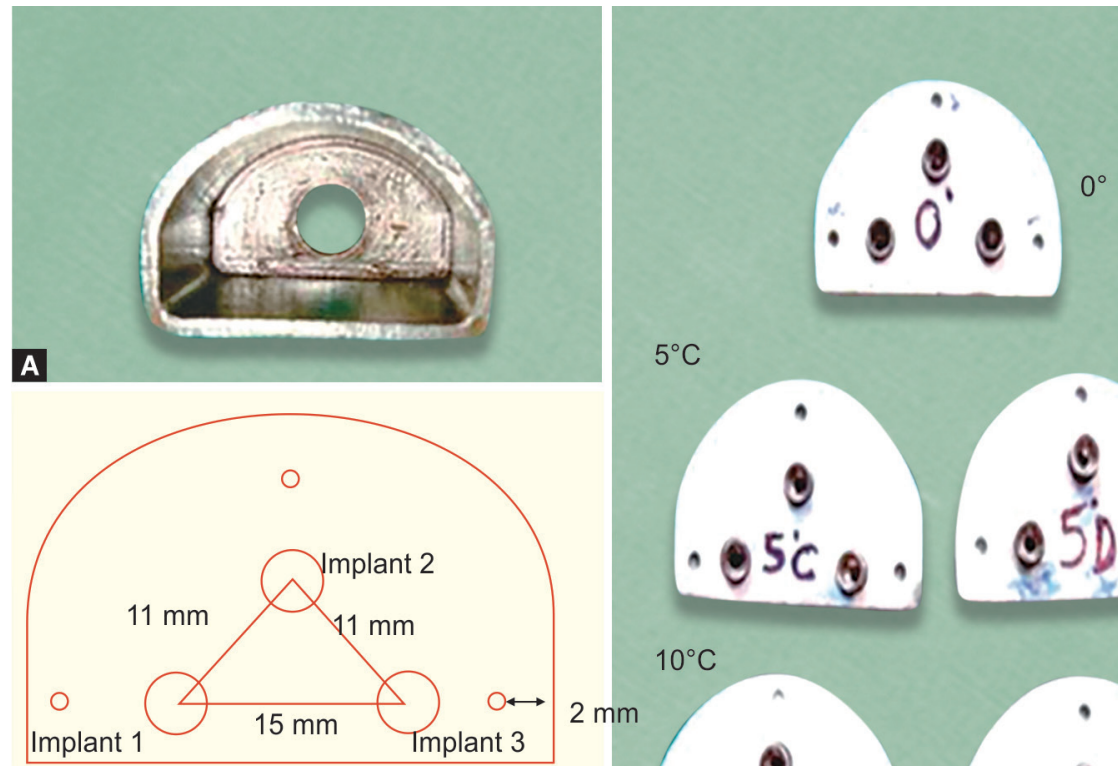

B

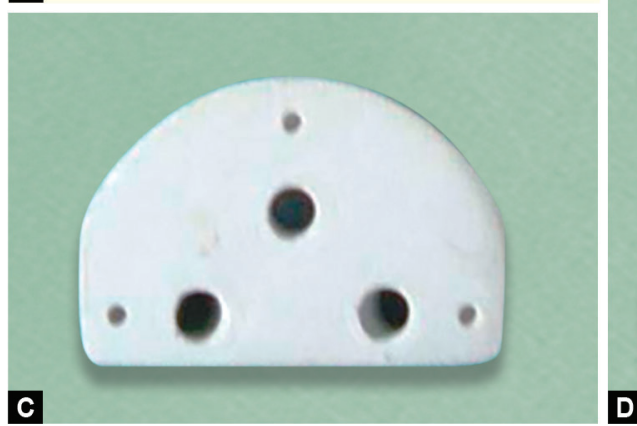

$10^{\circ} \mathrm{C}$
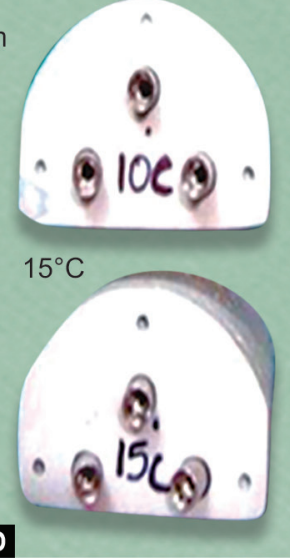

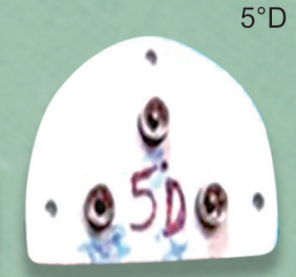

$5^{\circ} \mathrm{D}$
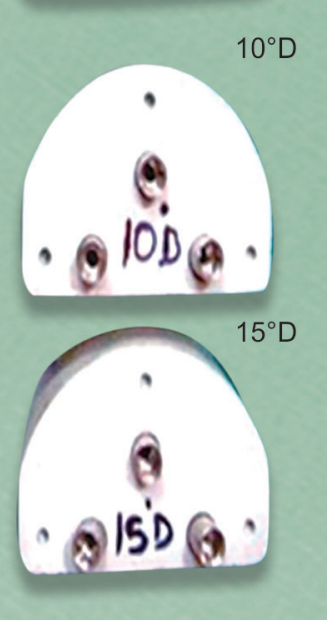

Figs 1 A to D: (A) Mold; (B) Schematic representation of the placement of implant analogs in a triangular pattern; (C) Holes punched in the cast; (D) Definitive casts 
Group 4: Implant numbers 1 and 3 are at $10^{\circ}$ convergence to the center implant (implant number 2).

Group 5: Implant numbers 1 and 3 are at $10^{\circ}$ divergence to the center implant (implant number 2).

Group 6: Implant numbers 1 and 3 are at $15^{\circ}$ convergence to the center implant (implant number 2).

Group 7: Implant numbers 1 and 3 are at $15^{\circ}$ divergence to the center implant (implant number 2).

Implant analogs were secured in all definitive casts with cyanoacrylate.

\section{IMPRESSION TRAYS AND IMPRESSION MAKING}

Three open tray impressions of definitive casts were made in each group, making a total of 21 impressions. A double layer of modeling wax was adapted on each of the definitive cast and a custom tray was fabricated with a light cure hybrid composite resin (Plaque photo, LiWa Light). Three openings were created in each and every custom tray corresponding to the implant analogs on the definitive casts for accommodating guide pins.

The trays were polymerized in the ultraviolet (UV) light cure unit (LiWa Light; Fig. 2A), trimmed, and finished. Placement of three open tray impression copings (Life Care Devices Pvt. Ltd.) was done on the three implants for transferring angulation and position of implant analogs. The copings engaged the hex and were tightened with a hex driver (Life Care Devices Pvt. Ltd.).

Vinyl polysiloxane adhesive (universal) was painted on custom trays and let dry for 15 minutes. One-step putty-wash impression was made of a definitive cast. For filling up the custom trays, heavy body vinyl polysiloxane impression material was used. On the definitive casts, light body vinyl polysiloxane impression material (Examix, GC) was injected around the impression copings.
Afterward, custom trays were placed and approximated on definitive casts. Using a finger swipe, excess material was removed from the open tray windows and guide pins were exposed. After 10 minutes (polymerization time of the impression material), loosening of the guide pins was done with the help of a hex driver. Removal of custom trays was done from the definitive casts. Connection of the three impression copings was established to the three implant analogs. This was followed by tightening of the guide pins with the hex driver (Fig. 2B).

With the help of wax, boxing of the impressions was done. Type IV dental stone (Zhermack) was manipulated and poured into the impression using a vibrator (Confident) and allowed to set for 1 hour.

The guide pins were loosened and separation of the impression from the casts was done. The separated casts were trimmed and labeled for analysis (Fig. 2C).

\section{Measurements}

Securing of both the casts was done on a mounting plate on the coordinate-measuring machine (Mitutoyo, Japan). A fine tip measuring stylus was used for assessing the coordinates on the implant (Fig. 3). The stylus was manually positioned on the implant analog top surface (six points) and the base of the cast (three points). Figure 4 shows the graphical representation of scanning data by the coordinate-measuring machine. On a datasheet, recording of the coordinative points was done with the purpose of aligning duplicate casts. With the help of algebra (vector), along the long axis of implant analog a vector was drawn in a parallel direction. Calculation of angular differences between the implant analog vectors was done from both the casts.

Repeated measures analysis of variance $(p=0.05)$ was the primary analysis. The duplicate cast was the "subject."
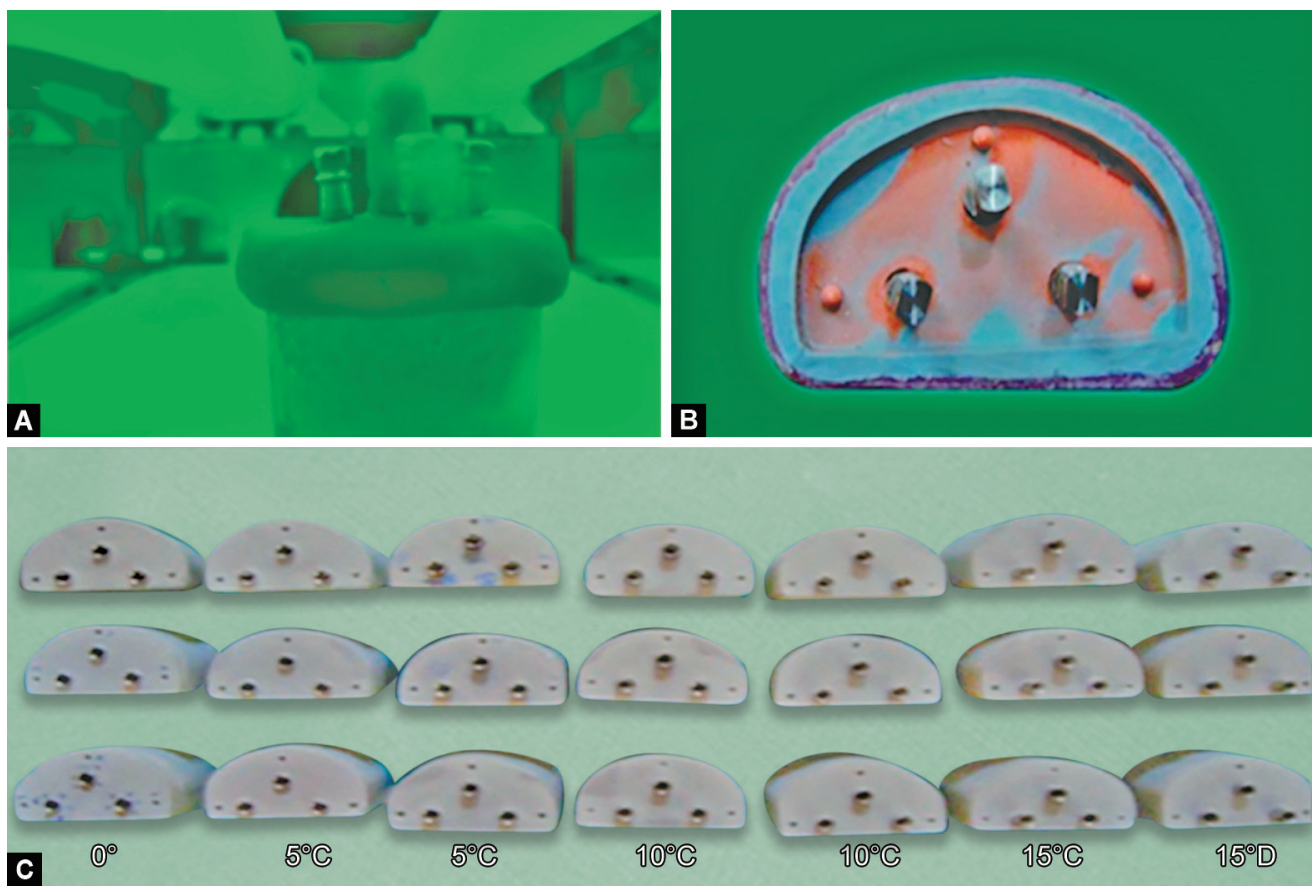

Figs 2A to C: (A) Impression tray being cured in the UV light cure unit (LiWa Light); (B) Final impression with the analogs attached to the impression copings; (C) Duplicate casts 
Implant number and the interaction with angulation of implant were the "within-subject" fixed effects.

Then, a post hoc analysis (using least significant difference) was done to study intergroup comparisons.

\section{Results}

Tables 1 to 4 show the results for implants $\left({ }^{\circ}\right)$ for different groups. While analyzing the impact of dental implant number and the correlative effect between dental implant number and dental implant angulation,

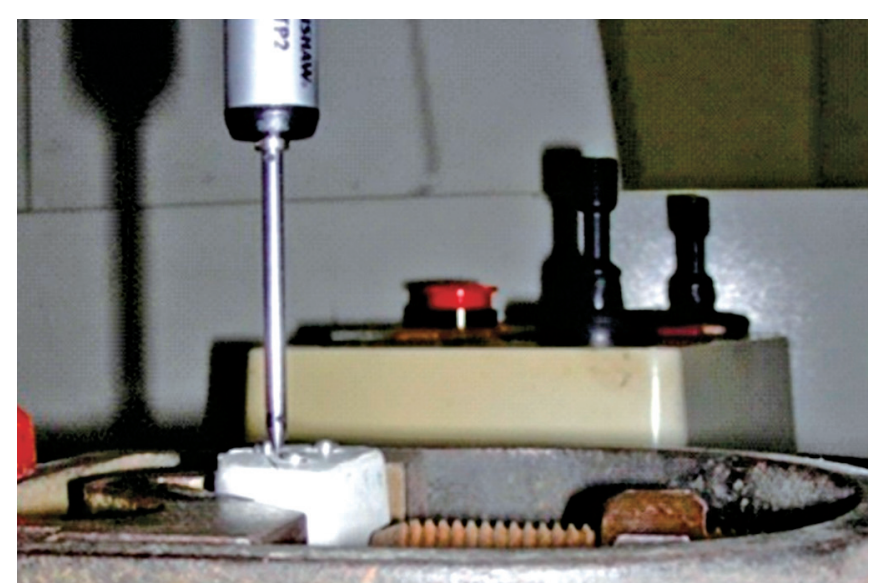

Fig. 3: Coordinates being measured on the cast significant results were obtained. However, nonsignificant results were obtained while evaluating the impact of dental implant angulation as shown in Tables 1 and 2. Significant results were obtained while comparing the effect of parallel group from $5^{\circ}$ divergence and $10^{\circ}$ convergence groups as shown in Table 3 and 4 . Also, significant results

Table 1: Effect of implant number and angulation on accuracy

\begin{tabular}{|c|c|c|c|c|}
\hline Effect & $\begin{array}{l}\text { Degree of } \\
\text { freedom }\end{array}$ & $\begin{array}{l}\text { Mean } \\
\text { square }\end{array}$ & Fratio & "p" value \\
\hline $\begin{array}{l}\text { Main effect of implant } \\
\text { angulation }\end{array}$ & 6 & 0.247 & 2.027 & 0.065 \\
\hline $\begin{array}{l}\text { Main effect of implant } \\
\text { number }\end{array}$ & 2 & 1.287 & 10.539 & $<0.001^{* *}$ \\
\hline $\begin{array}{l}\text { Interaction between implant } \\
\text { number and angulation }\end{array}$ & 12 & 0.380 & 3.111 & $<0.001^{* *}$ \\
\hline
\end{tabular}

${ }^{* *} p<0.001$, highly significant

Table 2: Comparison of seven implant angulations separately for each implant number

\begin{tabular}{llll}
\hline Implant number & Degree of freedom & Fstatistic & "p" value \\
\hline Implant 1 & 6 & 2.705 & $0.022^{*}$ \\
Implant 2 & 6 & 2.089 & 0.069 \\
Implant 3 & 6 & 3.972 & $0.002^{* *}$ \\
\hline
\end{tabular}

${ }^{*} p<0.05$, significant at $5 \%$ significance level; ${ }^{* *} p<0.01$, significant at $1 \%$ significance level

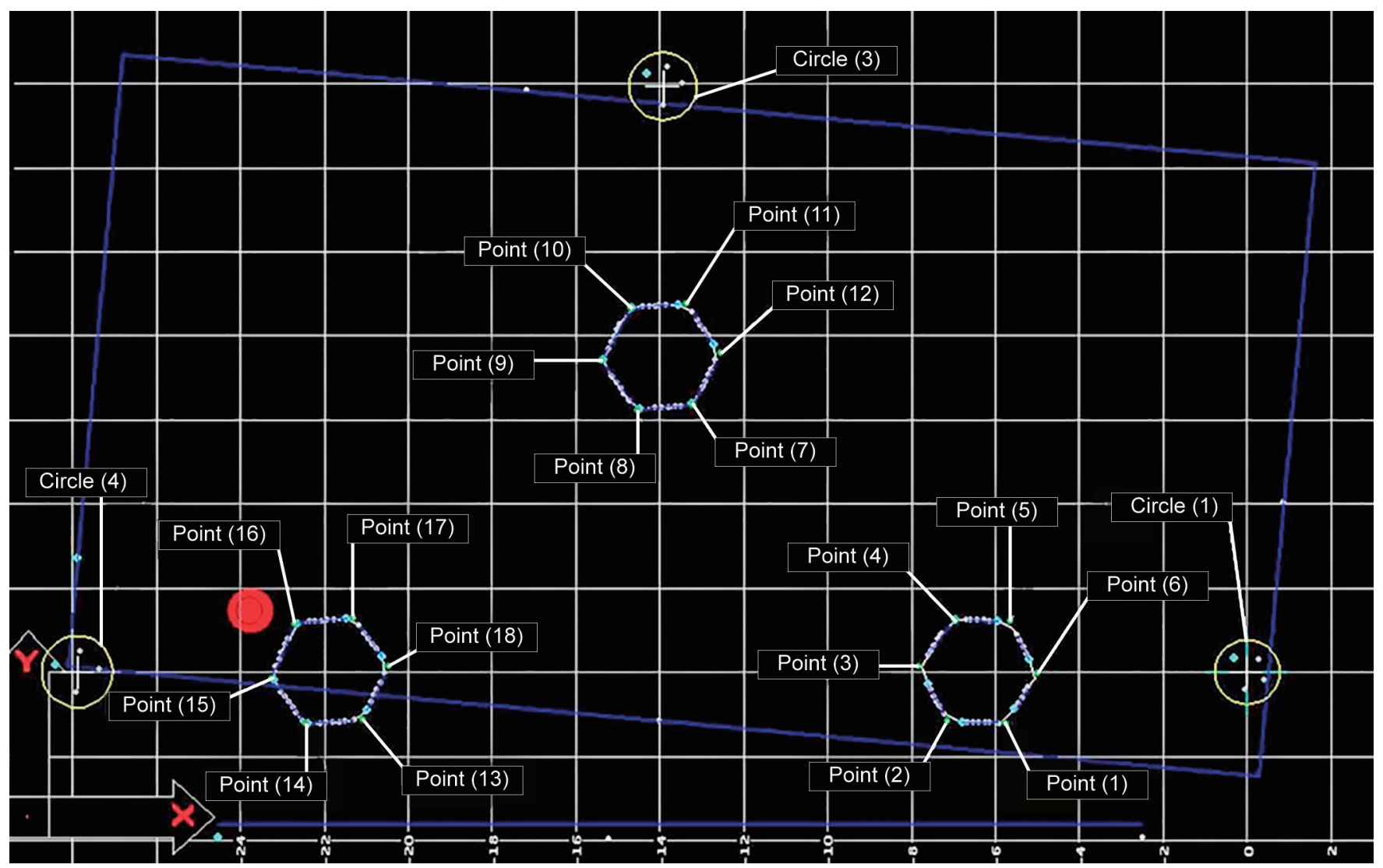

Fig. 4: Graphical representation of scanning of the cast by coordinate-measuring machine 
Table 3: Comparison of three implant numbers separately for each implant angulation

\begin{tabular}{lllcc}
\hline Implant angulation & $\begin{array}{l}\text { Degree of } \\
\text { freedom }\end{array}$ & $\begin{array}{l}\text { Mean } \\
\text { square }\end{array}$ & Fstatistic & " $p$ " value \\
\hline $15^{\circ}$ divergence & 2 & 0.251 & 2.590 & 0.096 \\
$10^{\circ}$ divergence & 2 & 0.863 & 5.122 & $0.014^{*}$ \\
$5^{\circ}$ divergence & 2 & 0.116 & 0.914 & 0.414 \\
Parallel & 2 & 0.034 & 0.546 & 0.586 \\
$5^{\circ}$ convergence & 2 & 1.291 & 8.758 & $<0.001^{* *}$ \\
$10^{\circ}$ convergence & 2 & 0.931 & 13.096 & $<0.001^{* *}$ \\
$15^{\circ}$ convergence & 2 & 0.079 & 0.436 & 0.652 \\
\hline${ }^{*} p<0.05$, significant at $5 \%$ significance level; ** $p<0.001$, highly significant
\end{tabular}

Table 4: Post hoc analysis (using LSD)_intergroup comparison

\begin{tabular}{|c|c|c|c|c|}
\hline Group & & $\begin{array}{l}\text { Mean } \\
\text { difference }\end{array}$ & S.E. & Significance \\
\hline \multirow[t]{6}{*}{ Parallel } & $5^{\circ}$ convergence & 0.1672 & 0.09509 & 0.080 \\
\hline & $5^{\circ}$ divergence & $0.2380^{*}$ & 0.09509 & 0.013 \\
\hline & $10^{\circ}$ convergence & $0.2014^{*}$ & 0.09509 & 0.036 \\
\hline & $10^{\circ}$ divergence & 0.1299 & 0.09509 & 0.174 \\
\hline & $15^{\circ}$ convergence & 0.0242 & 0.09509 & 0.800 \\
\hline & $15^{\circ}$ divergence & 0.0255 & 0.09509 & 0.789 \\
\hline \multirow{5}{*}{$\begin{array}{l}5^{\circ} \\
\text { convergence }\end{array}$} & $5^{\circ}$ divergence & 0.0708 & 0.09509 & 0.458 \\
\hline & $10^{\circ}$ convergence & 0.0342 & 0.09509 & 0.720 \\
\hline & $10^{\circ}$ divergence & -0.0374 & 0.09509 & 0.695 \\
\hline & $15^{\circ}$ convergence & -0.1431 & 0.09509 & 0.134 \\
\hline & $15^{\circ}$ divergence & -0.1417 & 0.09509 & 0.138 \\
\hline \multirow[t]{4}{*}{$5^{\circ}$ divergence } & $10^{\circ}$ convergence & -0.0366 & 0.09509 & 0.701 \\
\hline & $10^{\circ}$ divergence & -0.1082 & 0.09509 & 0.257 \\
\hline & $15^{\circ}$ convergence & $-0.2139 *$ & 0.09509 & 0.026 \\
\hline & $15^{\circ}$ divergence & $-0.2125^{*}$ & 0.09509 & 0.027 \\
\hline $10^{\circ}$ & $10^{\circ}$ divergence & -0.0716 & 0.09509 & 0.453 \\
\hline \multirow[t]{2}{*}{ convergence } & $15^{\circ}$ convergence & -0.1773 & 0.09509 & 0.064 \\
\hline & $15^{\circ}$ divergence & -0.1759 & 0.09509 & 0.066 \\
\hline \multirow[t]{2}{*}{$10^{\circ}$ divergence } & $15^{\circ}$ convergence & -0.1057 & 0.09509 & 0.268 \\
\hline & $15^{\circ}$ divergence & -0.1044 & 0.09509 & 0.274 \\
\hline $\begin{array}{l}15^{\circ} \\
\text { convergence }\end{array}$ & $15^{\circ}$ divergence & 0.0013 & 0.09509 & 0.989 \\
\hline
\end{tabular}

were obtained while comparing between the $5^{\circ}$ divergence group with $15^{\circ}$ convergence and $15^{\circ}$ divergence groups, respectively. No interpretable pattern was observed while analyzing these interactions. From these results, it can be inferred that duplicate casts of significant accuracy can be framed using an open tray technique.

\section{Discussion}

The term passive fit, with regard to the relationship of a prosthetic superstructure to its underlying implant abutments, appears with increasing regularity in the literature. As yet, no definition or parameters have been established as to what constitutes a passive fit. The task is to create as accurate a fit as is clinically possible to avoid bone strain resulting from uncontrolled loading of the implants through the superstructure. ${ }^{9-11}$
The accuracy of a master cast for treatment utilizing implants is of paramount importance. Any dimensional inaccuracy in this process will lead to a compromised result and possibly treatment failure. Therefore, the impression technique is critical stage in this process. $^{12}$

The open tray and closed tray impression techniques have been advocated for the transfer of implant positions before the fabrication of the prosthesis. The accuracy of the implant cast depends on the type of impression material, ${ }^{13}$ the implant impression technique, ${ }^{11-17}$ die material accuracy, and the implant master cast technique. ${ }^{15,18}$

Several authors have compared direct and indirect

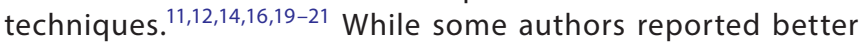
results with direct technique, others found the indirect technique more accurate.

Vigolo et al. found that direct splinted technique with acrylic splinting had better results than indirect technique or the nonsplinted direct techniques. ${ }^{21}$ Phillips and Inturregui suggested that the direct nonsplinted technique had better results because resin shrinkage due to polymerization can cause distortion. ${ }^{12,20} \mathrm{De}$ La Cruz et al. reported that the accuracy provided by verification jigs was not significantly superior to standard impression procedures. ${ }^{22}$

Many studies have shown that elastomeric impression materials, especially addition silicone and polyether, are most indicated for transfer impression procedures due to their increased linear stability, lower residual shrinkage during storage, greater rigidity, and lack of rotation resistance of coping inside the impression, leading to a more accurate cast. ${ }^{23,24}$

Wenz investigated different mixing methods of impression materials and found that the two-step vinyl polysiloxane impression was significantly less accurate than the one-step putty and light body vinyl polysiloxane combination impression, the medium body vinyl polysiloxane monophase impression, and the medium body polyether monophase impression. ${ }^{25}$

So in the present study, one-step putty-wash method was used with the addition of silicone putty and light body impression materials. Custom trays have been shown to produce more accurate implant-fixture level impressions than stock trays. ${ }^{26}$ Also, it has been shown that different thicknesses of elastomeric impression material can reduce accuracy, as the impression could shrink away from the impression specimen because of the material preferentially adhering to the tray rather than the specimen.

As light cure materials shrink less than self-cure acrylic resin, custom impression trays using light cure hybrid composite resin material were fabricated, providing double thickness of modeling wax as a spacer. Precise master cast fabrication is important in implant prosthodontics. Master casts poured in additional silicone and polyether with type IV die stone give better results which expands a similar amount as the impression material shrinks. ${ }^{27}$ So, in the present study type IV die stone was used to pour the casts.

In the present study, while analyzing the impact of dental implant number and the correlative effect between the number and angulation of dental implant, significant results were obtained. However, nonsignificant results were obtained while evaluating the impact of dental implant angulation. This implies clinically that an open tray technique can be used for making accurate casts for three implants, which are angled up to $15^{\circ}$.

Jemt and Book reported in their study that a certain biologic tolerance for a misfit may be present. ${ }^{28}$ The degree of misfit reported in the study was clinically acceptable with regard to observed marginal bone loss. 
This is in agreement with the studies conducted by Conrad et al. and Choi et al. who also reported accurate stability of open tray impressions with angulated implants. ${ }^{9,29}$ The study carried by Conrad et al. evaluated the accuracy of two implant-level impression techniques (direct nonsplinted and splinted). They concluded that the accuracy of implant-level impressions for internal-connection implant restorations was similar for the direct nonsplinted and splinted techniques in settings with divergence up to $8^{\circ} .^{29}$

Our results were, however, in discordance with the results obtained in the study conducted by Assuncao et al. who also evaluated the accuracy of the transfer process under variable conditions with regard to implant analog angulations- $90^{\circ}, 80^{\circ}$, $75^{\circ}$, and $65^{\circ}$ and different impression materials. It was concluded that the more perpendicular the implant analog angulation was in relation to the horizontal surface, the more accurate the impression was. ${ }^{23}$ The use of meticulous and accurate implant prosthodontic procedures is recommended as a means to attain the best possible fit of the implant prosthesis to the intraoral abutments. ${ }^{9}$

The results of the present study hold good for three implants angled up to $15^{\circ}$. It may not be as relevant for implant impressions having a lesser or more number of implants.

The final clinical goal is to manufacture an implant superstructure which divulges no stress on a completely seated dental implant. ${ }^{30}$

Our study had a few limitations. Firstly, it was an in vitro study. So, patient-related factors influencing the implant were not considered. Also, the number of implants was limited to three, and the angulation of implants was limited to $15^{\circ}$.

\section{Conclusion}

From the above results, it can be inferred that the close proximity of implant angulation toward the right-angled direction results in higher precision of implant. In the impression, there is direct picking from the impression copings which decreases the discrepancies in implant angulation on impression and master cast. Also, accurate implant impressions can be made using an open tray technique for three implants angled up to $15^{\circ}$.

\section{References}

1. Nicholson L. Transfer index of multiple angulated abutments in the restoration of an edentulous maxilla. J Prosthet Dent 1997;78(6):605608. DOI: 10.1016/s0022-3913(97)70012-x.

2. Rashidan N, Alikhasi M, Samadizadeh S, et al. Accuracy of implant impressions with different impression coping types and shapes. Clin Implant Dent Relat Res 2012;14(2):218-225. DOI: 10.1111/j.17088208.2009.00241.x.

3. Branemark PI. Osseointegration and its experimental background. J Prosthet Dent1983;50(3):399-410. DOI: 10.1016/s0022-3913(83)80101-2.

4. Jemt T, Rubenstein JE, Carlsson L, et al. Measuring fit at the implant prosthodontic interface. J Prosthet Dent 1996;75(3):314-325. DOI: 10.1016/s0022-3913(96)90491-6.

5. Rangert $B$, Jemt $T$, Jorneus $L$. Forces and moments on Branemark implants. Int J Oral Maxillofac Implants 1989;4(3):241-247.

6. Skalak R. Biomechanical considerations in osseointegrated prostheses. J Prosthet Dent 1983;49(6):843-848. DOI: 10.1016/00223913(83)90361-x.

7. Carr AB. Comparison of impression techniques for a five-implant mandibular model. Int J Oral Maxillofac Implants 1991;6(4):448-455.

8. Windhorn RJ, Gunnell TR. A simple open-tray implant impression technique. J Prosthet Dent 2006;96(3):220-221. DOI: 10.1016/j. prosdent.2006.07.009.
9. Conrad HJ, Pesun IJ, DeLong R, et al. Accuracy of two impression techniques with angulated implants. J Prosthet Dent 2007;97(6):349356. DOI: 10.1016/S0022-3913(07)60023-7.

10. Kohavi D. Combined impression technique for a partial implantsupported fixed-detachable restoration. Quintessence Int 1997;28:177-181.

11. Assif D, Marsha B, Schmidt A. Accuracy of implant impression techniques. Int J Oral Maxillofac Implants 1996;11(2):216-222.

12. Phillips KM, Nicholls Jl, Ma T, et al. The accuracy of three implant impression techniques: a three-dimensional analysis. Int J Oral Maxillofac Implants 1994;9(5):533-540.

13. Barrett MG, de Rijk WG, Burgess JO. The accuracy of six impression techniques for osseointegrated implants. J Prosthodont 1993;2(2):7582. DOI: 10.1111/j.1532-849x.1993.tb00387.x.

14. Carr AB. Comparison of impression techniques for a two-implant 15-degree divergent model. Int J Oral Maxillofac Implants 1992;7(4):468-475.

15. Hsu CC, Millstein PL, Stein RS. A comparative analysis of the accuracy of implant transfer techniques. J Prosthet Dent 1993;69(6):588-593. DOI: 10.1016/0022-3913(93)90287-x.

16. Humphries RM, Yaman P, Bloem TJ. The accuracy of implant master casts constructed from transfer impressions. Int J Oral Maxillofac Implants 1990;5(4):331-336.

17. Vigolo P, Fonzi F, Majzoub Z, et al. An evaluation of impression techniques for multiple internal connection implant prostheses. J Prosthet Dent 2004;92(5):470-476. DOI: 10.1016/j. prosdent.2004.08.015.

18. Caputi S, Varvara G. Dimensional accuracy of resultant casts made by a monophase, one-step and two-step, and a novel two-step putty/ light-body impression technique: an in vitro study. J Prosthet Dent 2008;99(4):274-281. DOI: 10.1016/S0022-3913(08)60061-X.

19. Burawi $G$, Houston F, Byrne D, et al. A comparison of the dimensional accuracy of the splinted and unsplinted impression techniques for the Bone-Lock implant system. J Prosthet Dent 1997;77(1):68-75. DOI: 10.1016/s0022-3913(97)70209-9.

20. Inturregui JA, Aquilino SA, Ryther JS, et al. Evaluation of three impression techniques for osseointegrated oral implants. J Prosthet Dent 1993;69(5):503-509. DOI: 10.1016/0022-3913(93)90160-p.

21. Vigolo P, Majzoub Z, Cordioli G. In vitro comparison of master cast accuracy for single-tooth implant replacement. J Prosthet Dent 2000;83(5):562-566. DOI: 10.1016/S0022-3913(00)70015-1.

22. De La Cruz JE, Funkenbusch PD, Ercoli C, et al. Verification jig for implant-supported prostheses: a comparison of standard impressions with verification jigs made of different materials. J Prosthet Dent 2002;88(3):329-336. DOI: 10.1067/mpr.2002.128070.

23. AssuncaoWG, Filho HG, Zaniquelli O. Evaluation of transfer impressions for osseointegrated implants at various angulations. Implant Dent 2004;13(4):358-366. DOI: 10.1097/01.id.0000144509.58901.f7.

24. Wee AG. Comparison of impression materials for direct multi-implant impressions. J Prosthet Dent 2000;83(3):323-331. DOI: 10.1016/s00223913(00)70136-3.

25. Wenz HJ, Hertrampf K. Accuracy of impressions and casts using different implant impression techniques in a multi-implant system with an internal hex connection. Int J Oral Maxillofac Implants 2008;23(1):39-47.

26. Burns J, Palmer R, Howe L, Wilson R. Accuracy of open tray implant impressions: an in vitro comparison of stock versus custom trays. J Prosthet Dent 2003;89(3):250-255. DOI: 10.1067/mpr.2003.38.

27. Misch CE. Contemporary implant dentistry. St Louis: Mosby; 1999.

28. Jemt T, Book K. Prosthesis misfit and marginal bone loss in edentulous implant patients. Int J Oral Maxillofac Implants 1996;11(5):620-625.

29. Choi JH, Lim YJ, Yim SH, et al. Evaluation of the accuracy of implantlevel impression techniques for internal-connection implant prostheses in parallel and divergent models. Int J Oral Maxillofac Implants 2007;22(5):761-768.

30. Adell R, Lekholm U, Rockler B, et al. A 15-year study of osseointegrated implants in the treatment of the edentulous jaw. Int Oral Surg 1981;10(6):387-416. DOI: 10.1016/s0300-9785(81)80077-4. 